\title{
A Small-Molecule Organic Semiconductor
}

\author{
(C) F. Yakuphanoglu, M. Aydin ${ }^{+}$, N. Arsu+ , M. Sekerci $\neq$ \\ Firat University, Faculty of Arts and Sciences, Department of Physics, \\ 23119 Elazig, Turkey \\ + Yildiz Technical University, Faculty of Arts and Sciences, Department of Chemistry, \\ 34210 Davutpaşa-İstanbul, Turkey \\ F Firat University, Faculty of Arts and Sciences, Department of Chemistry, \\ 23119 Elazig, Turkey
}

(Получена 7 августа 2003 г. Принята к печати 16 сентября 2003 г.)

The electrical conductivity and optical properties of the bis-diethylaminokumarin have been investigated. The electrical conductivity of the compound exhibited three-dimensional hopping conduction in the temperature range 295-321 K. The compound shows typical semiconductor property and its semiconducting property results from delocalization of the $\pi$-electrons in the structure. To determine the optical band gap of the compound, an optical absorption study was made in the wavelength range $250-600 \mathrm{~nm}$. The optical study revealed that the optical transition is the allowed indirect one. The electronic parameters such as electrical conductivity at room temperature $\sigma_{25}$, activation energy $E$, and optical band gap $E_{g}$, of the compound are $1.46 \cdot 10^{-5} \mathrm{~S} / \mathrm{cm}, 0.42 \mathrm{eV}$ and $2.46 \mathrm{eV}$, respectively. These values are in agreement with electronic parameters of organic semiconductors.

\section{Introduction}

The organic semiconducting materials are grouped as polymer, monomer, and organic compounds. Their current revival of interest in the electronic properties is reflected by a considerable increase in the number of investigations dealing with the measurement of electrical conductivity and they are of great interest in electronic devices and multiple advantages because of the variety of structures. Their electrical transport properties are of current interest and they give different aspects of the conduction mechanism, nature of charge carriers and their properties [1]. In the literature, the electrical properties of many semiconducting organic compounds are reported [1]. Intensive research activities on organic semiconducting materials have led to insights into the chemical and physical properties. The potential usefulness of semiconducting materials have been explored in many fields such as electronic switches, batteries, light emitting diodes and Schottky diodes [2,3]. They have attracted considerable attention, since the investigation of these systems has generated entirely new scientific conceptions and a potential for its perspective application in molecular electronics. They contain an extended $\pi$ electron system and this system can be changed from a semiconducting state to a conducting state [4].

Organic semiconductors are used extensively in the fabrication of active and passive solid-state devices. In this connection, we synthesized bis-diethylaminokumarin to create a semiconductor material and investigate its electrical and optical properties.

\section{Experimental}

Bis-diethylaminokumarin was synthesized according to the literature [5]. The chemical structure of the investigated compound is given in Fig. 1. The powders were compacted at pressures in the order of $10^{4} \mathrm{~kg} / \mathrm{cm}^{2}$. Fig. 2 shows the dia- gram of the electrical conduction measurement system. The conductivity measurements were performed on compacted powders $[5,6]$ using a Keithley $20006^{1 / 2}$ digital multimeter. To determine the optical properties of the compound, the UV spectra were recorded at room temperature with a Philips PU 8700 UV-Vis spectrophotometer.<smiles>CCCCNC1=CC2OC(=O)C(=O)C(=O)C2C=C2C=CC(N(CC)CC)=CC2C=C1</smiles>

Figure 1. The chemical structure of the compound.

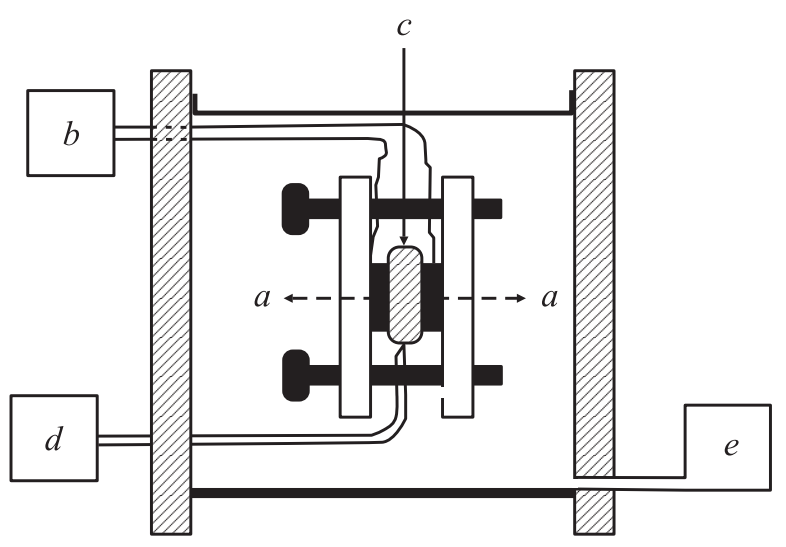
$a$ : electrodes
$b$ : digital multimeter
$c$ : sample
$d$ : thermocouple controller
$e$ : heating and cooling system

Figure 2. The experimental setup for electrical conductivity measurements. 
Table 1. The electronic parameters of the compound

\begin{tabular}{c|c|c|c|c|c|c|c}
\hline$E(\mathrm{eV})$ & $\sigma_{25}(\mathrm{~S} / \mathrm{cm})$ & $\sigma_{0}(\mathrm{~S} / \mathrm{cm})$ & $\lambda_{\max }(\mathrm{nm})$ & $E_{g}(\mathrm{eV})$ & $E_{0}(\mathrm{meV})$ & $n($ concentration $)$ & Molar absorption coefficient $(\varepsilon)$ \\
\hline 0.42 & $1.46 \cdot 10^{-5}$ & 145.77 & 459 & 2.46 & 46.4 & $1.2755 \cdot 10^{-5} \mathrm{~mol} / 1$ & $860001 /(\mathrm{mol} \cdot \mathrm{cm})$
\end{tabular}

\section{Results and discussion}

\subsection{DC conductivity}

The temperature dependence of the conductivity is shown in Fig. 3. With temperature, the conductivity of the compound substantially increases up to $321 \mathrm{~K}$ (I region) and afterwards, the conductivity is decreased (II region). The linear portion of the graph (region I) is fitted to an Arrhenius type equation [6],

$$
\sigma=\sigma_{0} \exp (-E / k T),
$$

where $\sigma_{0}$ is a constant, $E$ is the activation energy. The electronic parameters, $\sigma_{0}$ and $E$ were calculated and are given in Table 1 . In region I, the conductivity increases with increasing temperature due to delocalized $\pi$-electrons.

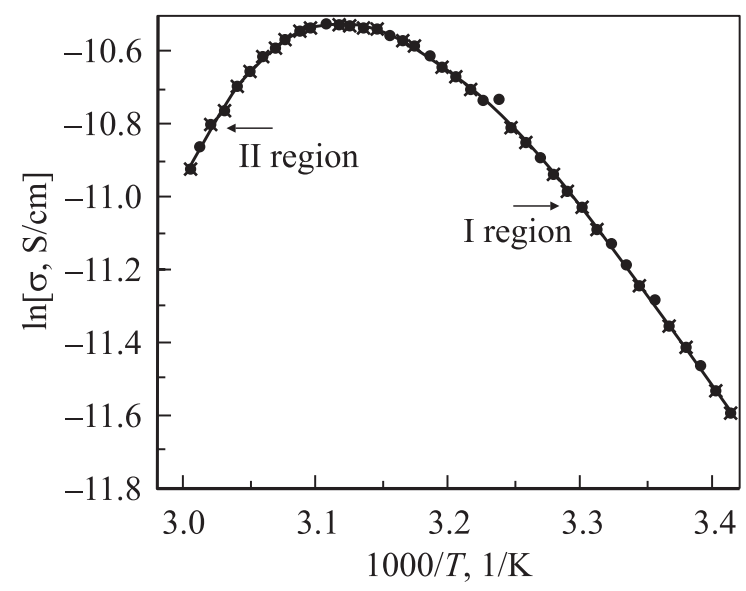

Рис. 3. The electrical conductivity of the compound.

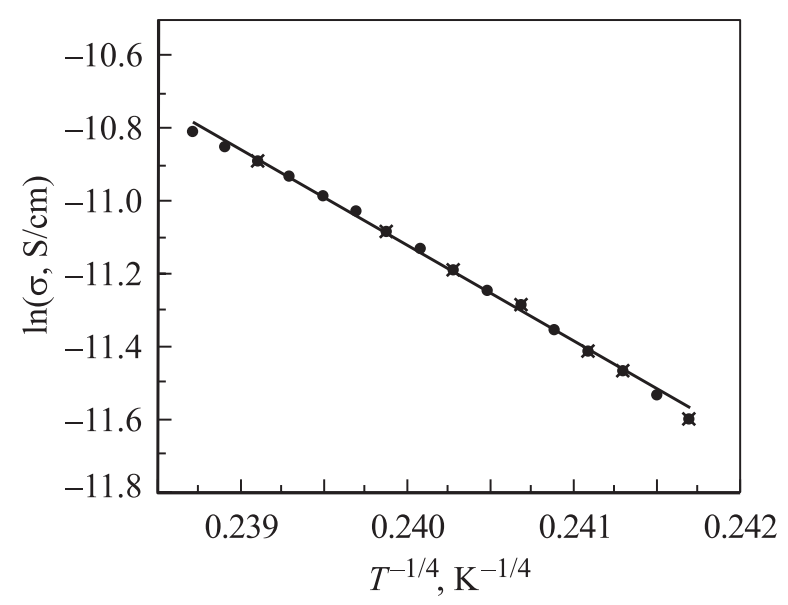

Puc. 4. The three-dimensional hopping conduction of the compound.
The exponential band tail in the compound as observed us from UV absorption spectra of the compound indicated that there are localized states, which lead to variable hopping conduction (VRH). Thus, conduction mechanism for the first region, can be expressed by the following equations [7],

$$
\sigma(T)=\sigma_{1} \exp \left[-\left(\frac{T_{0}}{T}\right)^{1 / 4}\right]
$$

This behavior is referred to as variable-range hopping conduction by Mott. We have applied the VRH model to investigate conduction mechanism of this compound. For this purpose, we fitted our conductivity data to Eq. (2) using a least-square fit procedure so that the best fit values of electronic parameters may be obtained. The $\ln \sigma$ vs $T^{1 / 4}$ was plotted and is shown in Fig. 4. In Eq. (2), $T_{0}$ can be expressed by the following relation [7],

$$
T_{0}=\frac{16}{N\left(E_{\mathrm{F}}\right) k L_{\mathrm{loc}}^{3}}
$$

and

$$
R=\left[\frac{9 L_{\mathrm{loc}}}{8 \pi k T N\left(E_{\mathrm{T}}\right)}\right]^{1 / 4},
$$

where $L_{\text {loc }}$ is the localization length, $N\left(E_{\mathrm{F}}\right)$ is the density of states at the Fermi level, $R$ is the hopping distance. Assuming $L_{\mathrm{loc}}=10^{-7} \mathrm{~cm}$ [7], we calculated the electronic parameters of the compound using these relations and data given in Table 2. It is found that this compound exhibits three-dimensional hopping conduction in the temperature range $295-321 \mathrm{~K}$. In this conduction mechanism, when the temperature is low enough so that carriers cannot be excited into one of allowed bands, the dominant conduction takes place via hopping conduction of the charge carriers in the localized states near the Fermi level [7].

Таблица 2. The hopping conduction parameters of the compound

\begin{tabular}{c|c|c}
\hline$T_{0}(\mathrm{~K})$ & $N\left(E_{\mathrm{F}}\right)\left(\mathrm{m}^{-3} \mathrm{eV}^{-1}\right)$ & $R(\mathrm{~m})$ \\
\hline $4.74 \cdot 10^{9}$ & $4.66 \cdot 10^{22}$ & $2.33 \cdot 10^{-8}$
\end{tabular}

As seen in Fig. 3, in region II, a deviation from the Arrhenius equation (1) appears within the temperature range $T>321 \mathrm{~K}$ the conductivity decreases with temperature. The thermal variation of $\sigma(T)$ can be attributed to the saturation of the electronic concentration growth, typical for semiconductors when all carriers are released from the localization centers to the conduction 


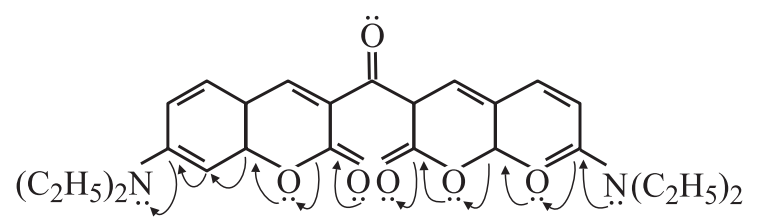

Рис. 5. The possible delocalization of $\pi$-electrons in structure of the compound.

band. Subsequent decrease of the conductivity, again similarly to semiconductor behavior, can result form the decrease of the carrier mobility caused by the scattering no the lattice vibrations.

This discussion shows that the compound is a semiconductor and has semiiconducting properties in the temperature region under consideration. This type of conduction agrees with those of usual organic compounds. The conductivity of the compound could be attributed to thermal excitations of the carbon-oxygen bonds in the structure. The lone pair of electrons on the oxygen atom with the $\sigma$-bond in the structure makes the delocalization of the $\pi$-electrons possible, as shown in Fig. 5.

It is well known that the electronic transport properties of organic semiconductors depend on their chemical structure [4]. It is seen from the chemical structure of the compound that there are many $\pi$-electrons in the structure due to the aromatic groups. In such a structure large numbers of electrons cause a decrease of the excitation energy of the $\pi$-electrons. The $\pi$-electrons create also a decrease in the system's internal energy and apparently allow electrons to participate in conduction. The increase of the conductivity in region I at $T<321 \mathrm{~K}$ (see Fig. 4) can be associated with the number of $\pi$-electrons in the compound.

As result, the conduction may be explained according to the chemical structure of the compound: it is seen from Fig. 1 that in the structure benzene rings are available which have many $\pi$-electrons. The $\pi$-electrons are mobile in the structure and thus, conduction results from the transfer of $\pi$-electrons. The electronic excitation of the benzene rings followed by transfer of an electron to another molecule is considered to be an important step of the conduction process. The studied compound possesses $\pi$-electrons extending along the structure, its electron states are delocalized, allowing the electronic transport to be described in terms of hopping conduction.

\subsection{Optical properties}

The UV spectrum of the bis-diethylaminokumarin was recorded in the wavelength range of $250-600 \mathrm{~nm}$ and is given in Fig. 6. Some parameters related to the spectrum are given in Table 1. The nature of the optically induced transitions can be determined from the UV spectrum. The relation between the absorption coefficient $\alpha$, and optical band gap $E_{g}$, is given by the following relation [8]

$$
\alpha h v=B\left(h v-E_{g}^{\mathrm{opt}}\right)^{2},
$$

where $B$ is an energy-independent constant and $E_{g}$ is the indirect optical band gap. The optical band gap of the compound was calculated by extrapolating the straight line portions of the $(\alpha h v)^{1 / 2}$ vs $h v$ curve (Fig. 7) and is given in Table 1. The indirect forbidden gap determined by the optical method is larger than $2 E$ obtained from the electrical conductivity measurements. Thus, it is evaluated that the electronic activation energy, $\Delta E$, may correspond to any electronic level in the band gap of the compound.

The optical absorption coefficient near the fundamental absorption edge is found to be exponentially dependent on

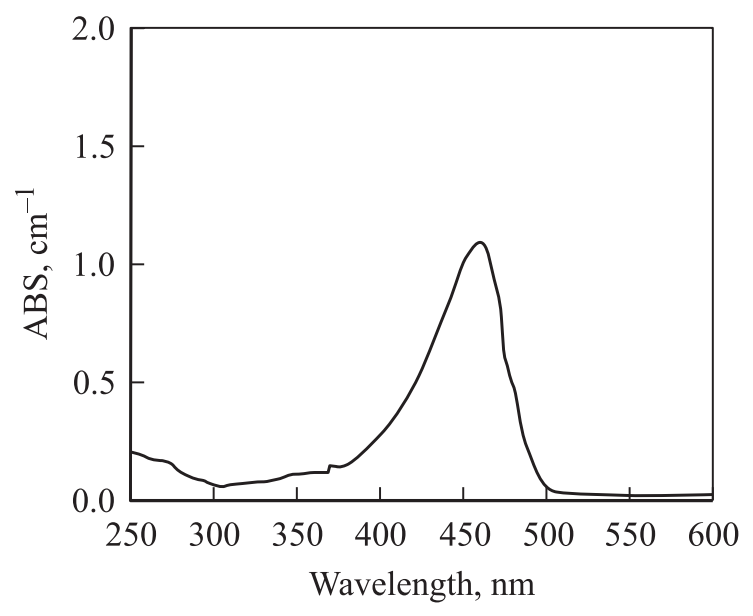

Рис. 6. UV spectrum of bis-diethylaminokumarin.

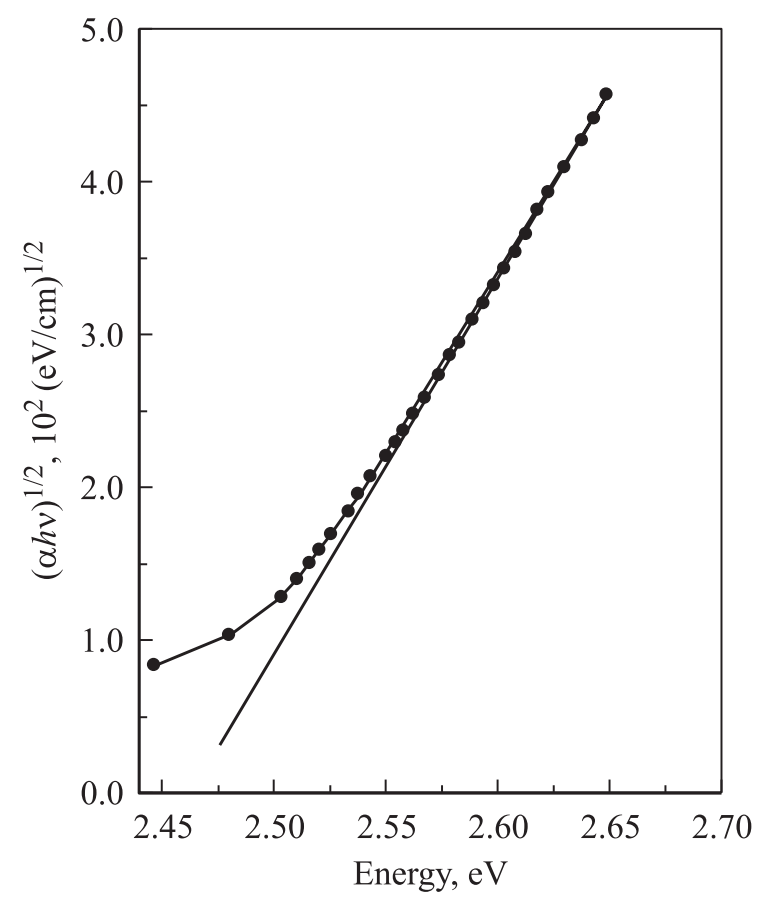

Pиc. 7. Extrapolation to zero of the curve representing the function $(\alpha h v)^{1 / 2}=f(h v)$. 


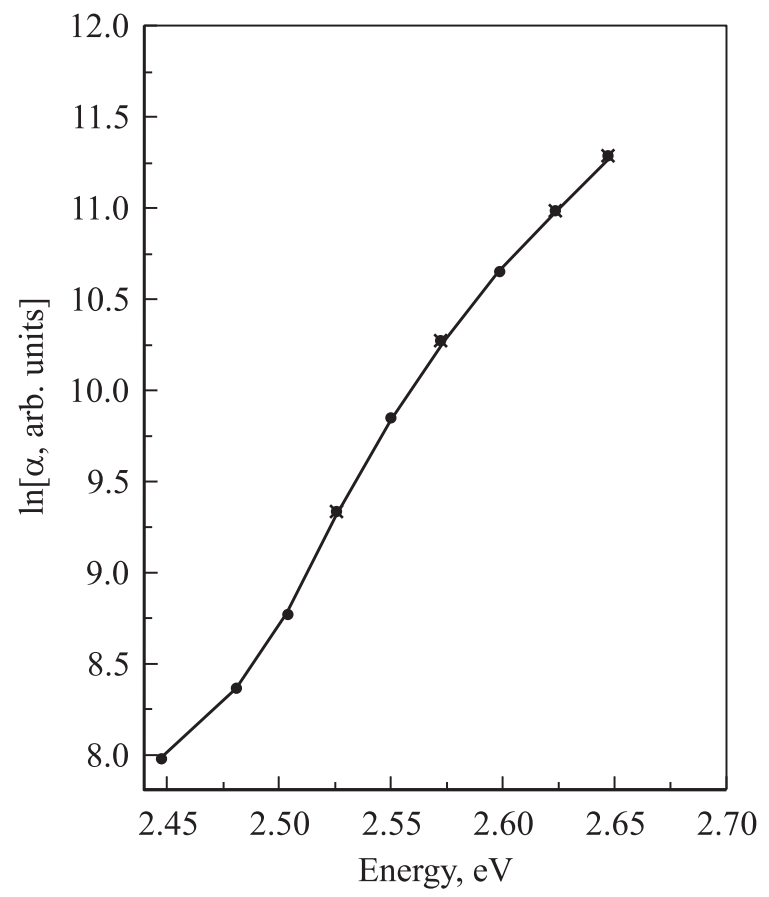

Pис. 8. Plot of $\ln \alpha$ vs $h v$ of the compound.

the incident photon energy and obeys the empirical Urbach rule $[9]$

$$
\alpha=\alpha_{0} \exp \left(\frac{h v}{E_{0}}\right)
$$

where $E_{0}$ is the Urbach energy and it can be evaluated as the width of the localized states [6]. The variation of $\ln \alpha$ vs photon energy is given in Fig. 8. The value of $E_{0}$ was calculated from Fig. 8 and is given in Table 1. The exponential dependence of $\alpha$ on photon energy for the compound indicates that it obeys Urbach's energy equation. An electronic transition between localized states in the band edge tails is valid in this compound. The exponential dependence of the optical absorption coefficien with photon energy may arise from the electronic transitions between the localized states, which tail off in the band gap [10].

\section{Conclusions}

The electrical conductivity and optical properties of the bis-diethylaminokumarin have been investigated. It is found that the compound is a typical organic semiconductor with calculated electronic parameters.

\section{References}

[1] R.V. Rao, M.H. Shridhar, S. Ganesh, K.C. Prashanth. Chem. Phys. Lett., 341, 306 (2001).

[2] G.D. Sharma, D. Saxena, M.S. Roy. Synt. Met., 123, 189 (2001).

[3] Extended Linear Chain Compounds, ed. by J.S. Miller (N.Y., Plenum Press, 1983) v. I-III.
[4] J.P. Farges. Organic Conductors (N.Y., 1994).

[5] D.P. Specht. P.A. Martic, S. Farid. Tetrahedron, 38, 1203 (1982).

[6] F. Yakuphanoglu. Ph.D. Thesis. Firat University (Elazig, Turkey, 2002).

[7] B.I. Shklovskii, A.L. Efros. Electronic Properties of Doped Semiconductors (Berlin, Springer, 1984).

[8] H. Hasegawa, S. Yazaki, T. Shimizu. Sol. St. Commun., 26, 4070 (1978).

[9] F. Urbach. Phys. Rev., 92, 627 (1966).

[10] E. Abd El-Wahabb, M.M. El-Samandouy, M. Fadel. Appl. Surf. Sci., 174, 106 (2001).

Редактор Т.А. Полянская 